\title{
IGF2/H19 hypomethylation in a patient with very low birthweight, preocious pubarche and insulin resistance
}

\author{
Rinki Murphy ${ }^{1 *}$, Lourdes Ibáñez ${ }^{2}$, Andrew Hattersley ${ }^{3}$ and Jörg Tost ${ }^{4}$
}

\begin{abstract}
Background: Insulin like growth factor 2 (IGF2) is an imprinted gene, which has an important role in fetal growth as established in mice models. IGF2 is downregulated through hypomethylation of a differentially methylated region (DMR) in Silver Russell syndrome (SRS), characterised by growth restriction. We have previously reported that severe pre- and post-natal growth restriction associated with insulin resistance and precocious pubarche in a woman without body asymmetry or other SRS features resulted from a balanced translocation affecting the regulation of her IGF2 gene expression. We hypothesised that severe pre- and post-natal growth restriction associated with insulin resistance and precocious pubarche in the absence of SRS are also caused by downregulation of IGF2 through hypomethylation, gene mutation or structural chromosomal abnormalities.

Methods: We performed routine karyotyping, IGF2 gene sequencing and investigated DNA methylation of the IGF2 differentially methylated region (DMR)0 and H19 DMR using pyrosequencing, in four women selected for very low birth weight ( $<-3$ SDS for gestational age), precocious pubarche, short adult stature $(<-2$ SDS), and insulin resistance (defined as HOMA-IS $<80 \%$ ); and compared their methylation results to those of 95 control subjects.

Results: We identified a 20 year old woman with severe hypomethylation at both DMRs. She was the smallest at birth (birthweight SDS,-3.9), and had the shortest adult height $(143 \mathrm{~cm})$. The patient was diagnosed with polycystic ovarian syndrome at the age of 15 years, and had impaired fasting glucose in the presence of a low BMI $\left(19.2 \mathrm{~kg} / \mathrm{m}^{2}\right)$.

Conclusions: Our case of growth restriction, premature pubarche and insulin resistance in the absence of body asymmetry or other features of SRS adds to the expanding phenotype of IGF2/H19 methylation abnormalities. Further studies are needed to confirm whether growth restriction in association with premature pubarche and insulin resistance is a specific manifestation of reduced IGF2 expression.
\end{abstract}

Keywords: Insulin like growth factor 2, Intrauterine growth restriction, Short stature, Insulin resistance

\section{Background}

Aberrant imprinting of the IGF2/H19 locus on chromosome 11p15.5 has a crucial role in Beckwith-Wiedemann syndrome (BWS, OMIM \#130650) [1], Silver-Russell syndrome (SRS, OMIM\#180860) [2,3] and several human cancers [4,5]. Paternal IGF2 and maternal H19 expression in humans, correlates with the close regulation in mice, by two differentially methylated regions (DMRs).

\footnotetext{
*Correspondence: R.Murphy@auckland.ac.nz

${ }^{1}$ Faculty of Medical and Health Sciences, University of Auckland, Auckland Private Bag 92019, New Zealand

Full list of author information is available at the end of the article
}

Both are normally methylated on the paternally inherited allele: IGF2 DMR0 (located between exons 2 and 3 of IGF2), and H19 DMR, located $4 \mathrm{~kb}$ upstream of the transcription start site of $H 19$ [6], resulting in around 40-50\% methylation at each of these sites [7-9] (Figure 1 A). The H19 DMR locus is also known as the imprinting control region (ICR) of the IGF2-H19 locus because it is the centre from where methylation on the paternal allele is propagated, including the IGF2 DMR0, H19 promoter DMR (which directly silences H19 expression from the paternal allele), in addition to containing the recognition sites for CCCTC binding factor (CTCF). CTCF is only

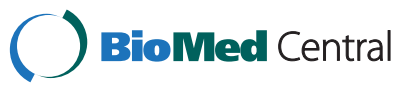



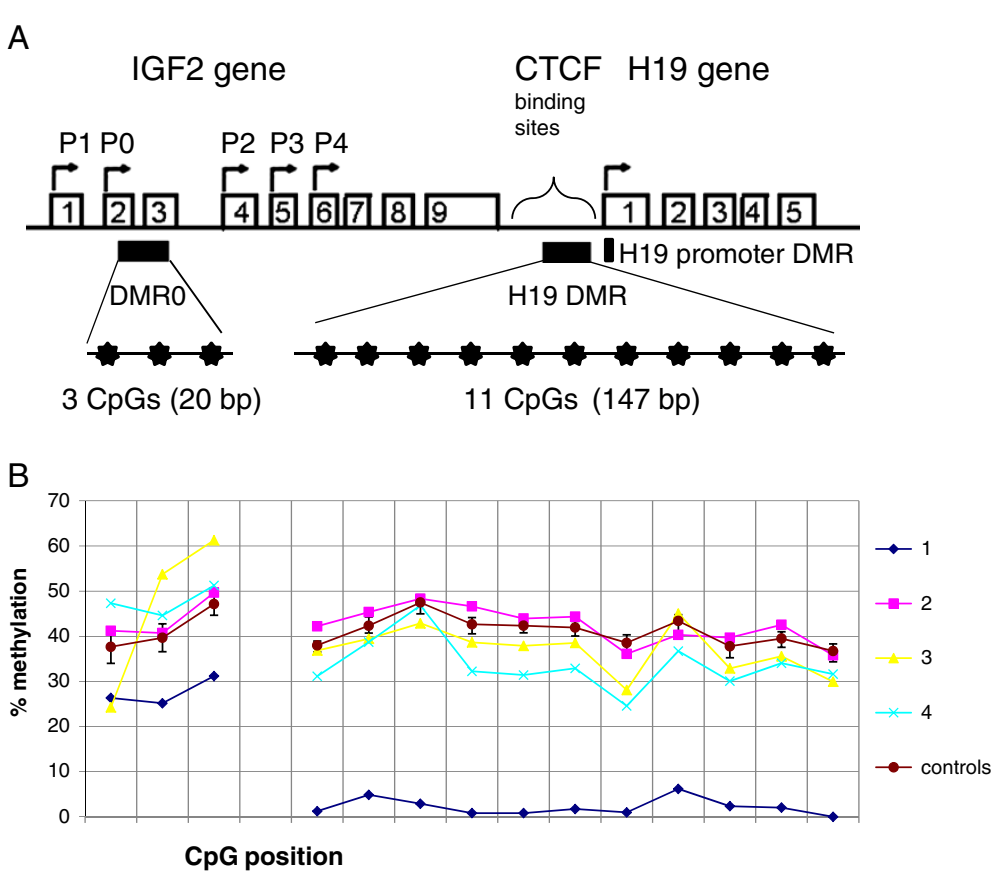

Figure 1 A Structural characteristics of the human IGF2/H19 locus with nine exons and five exons for IGF2 and H19 genes respectively. Transcription start site and promoters used are indicated by arrows. Regions of differentially methylated regions (DMRs) are shown with black bars. For each DMR, the number of CpGs analysed is indicated and their relative positions are represented by filled stars corresponding to the methylation site horizontal axis of Figure 1 B. B Graph of methylation patterns of the IGF2/H19 locus (expressed as percentage for each CpG) for each case and mean with $95 \%$ confidence interval for the 95 control samples.

able to bind to the unmethylated H19 DMR on the maternal allele which blocks the access of maternal allele IGF2 promoters from its downstream enhancers, but enables H19 expression from this allele [10].

Hypermethylation of the H19 DMR on the maternal allele associated with IGF2 over-expression is found in 10\% of patients with neonatal overgrowth and transient neonatal hypoglycemia characteristic of BWS [1], while hypomethylation of the H19 DMR on the paternal allele (associated with IGF2 under-expression) is found in over $50 \%$ of patients with SRS $[9,11]$, characterized by intrauterine and post-natal growth restriction. In a study of 70 BWS and 2 SRS patients, the methylation patterns at the IGF2 DMR0 resembled that at the H19 DMR, in contrast to 33 non-syndromic Wilms tumours in which IGF2 DMR0 methylation was negatively correlated with H19 DMR methylation [12]. Selective hypomethylation for either the H19 DMR or the IGF2 DMR0 has been reported in 13 patients with SRS [13]. The etiological role of IGF2 in regulating intrauterine growth and tumour formation has been established in animal studies $[14,15]$; however its role in the other features of BWS (such as exomphalos, macroglossia, ear abnormalities, hemihyperplasia) and SRS (craniofacial abnormalities, clinodactyly, genital abnormalities) is unknown, but might relate to the wide range of tissues in which IGF2 is expressed.
Besides imprinting abnormalities, structural chromosomal abnormalities affecting the IGF2/H19 locus have been described: Maternal 11p15 duplications (predicted to decrease IGF2 expression through increased doseage of maternal suppressor sequences) have been found in growth restricted patients [16,17], while paternal $11 \mathrm{p} 15$ duplications (predicted to increase IGF2 expression through relatively reduced doseage of maternal suppressor sequences) have been associated with BWS patients [1]. We previously reported a woman with severe intrauterine growth restriction (birthweight SDS -5.4), short stature and a balanced chromosomal translocation breakpoint $\mathrm{t}(1,11)(\mathrm{p} 36.22 ; \mathrm{p} 15.5)$, disrupting the access of her paternally inherited IGF2 allele from its telomeric enhancer sequences [18]. Although she was predicted to have paternal IGF2 under-expression similar to those with SRS, she did not fit the diagnostic criteria for SRS, beyond the obligatory pre- and post-natal growth restriction, including lack of any dysmorphisms supported by normal childhood photographs [18]. In addition, she had insulin resistance and precocious pubarche (pubic hair before the age of 8 yrs), two characteristics not commonly reported in association with SRS [18]. Due to the phenotype of this case, we sought to identify additional cases of paternal IGF2 inactivation through either structural chromosomal abnormalities, IGF2 gene mutations 
or IGF2/H19 methylation abberrations, by screening women with similar characteristics of intrauterine growth restriction, short stature, insulin resistance and precocious pubarche. Here we provide this data including detailed methylation patterns of both IGF2 DMR0 and H19 DMR in 4 women and in 95 control subjects from a healthy population.

\section{Methods}

\section{Participants}

The study population consisted of four girls (age, 13$20 \mathrm{yr}$ ) recruited from a tertiary Spanish hospital without any congenital abnormalities or dysmorphism, selected on the following criteria:

- Birthweight standard deviation score (SDS) below -3.0 after a term gestation not complicated by gestational diabetes, preeclampsia or use of alcohol or drugs;

- A history of precocious pubarche attributable to premature adrenarche, as judged by increased dehydroepiandrosterone-sulfate (DHEAS) and/or androstenedione levels for chronological age [19].

- Adult height SDS below -2.0 for Spanish population standards, with closed epiphysis.

- Decreased fasting insulin sensitivity, estimated from fasting insulin and glucose levels using the homeostasis model assessment (HOMA-CIGMA Calculator program, version 2.00), and defined as a HOMA-IS value less than $80 \%$ [20].

None of the patients had thyroid dysfunction or lateonset congenital adrenal hyperplasia; none were receiving any medication known to affect gonadal function or carbohydrate metabolism. At the time of the study, all were at least 2 yr beyond menarche (mean, 6.5 years).

In all cases, we undertook karyotyping analysis, screened for mutations in the IGF2 gene and performed methylation analysis of the H19 DMR, and IGF2 DMR0. The study also involved 95 control patients enrolled in the Exeter Family Study of Childhood Health (EFSOCH) [21] for comparative methylation analysis of the IGF2/ H19 locus.

\section{Ethical approval}

The study was approved by the Institutional Review Boards of the participating hospitals. Informed consent was obtained from parents and/or the subjects.

\section{Assessments}

Birth weight, birth length, gestational age and current height were obtained from hospital records, and SDS were derived from local reference values [22].
Serum glucose was measured by the glucose oxidase method. Immunoreactive insulin was assayed by IMX (Abbott Diagnostics), and serum sex hormone-binding globulin (SHBG), testosterone, androstenedione and DHEAS were assayed by immunochemiluminiscence (IMMULITE 2000, Diagnostic Products, Los Angeles, $\mathrm{CA}$ ), as described [23]; the intra- and inter-assay coefficients of variation (CVs) were between $4 \%$ and $8 \%$. The free androgen index, equivalent to free testosterone, was calculated as total testosterone/(nmol/L)/SHBG $(\mathrm{nmol} / \mathrm{L})$ $\mathrm{x} 100$. The samples were kept frozen at $-20^{\circ} \mathrm{C}$ until assay.

\section{DNA sequencing}

Leucocyte derived DNA from all study participants was sequenced for IGF2 mutations as previously described [18].

\section{DNA methylation analysis}

500 ng Genomic DNA (derived from peripheral blood leucocytes) was treated with sodium bisulphite and then $50 \mathrm{ng}$ of this DNA was used as a template for PCR amplification. The three DMRs were analyzed: IGF2 DMR0 (3 CpGs), and in the $3^{\text {rd }}$ CTCF binding site of the H19 DMR (11 CpGs) by pyrosequencing as previously described [24]. Accession numbers and nucleotide positions of each DMR, PCR primers and annealing temperatures as well as the size of PCR products have been detailed previously [25].

\section{Statistical analysis}

Methylation levels at IGF2 DMR0 (3 CpGs), and H19 DMR (11CpGs) in the four cases are graphed with corresponding median and IQR methylation levels of the 95 control EFSOCH samples (Figure 1B).

\section{Results}

SRS was not diagnosed in any of these women, based on absence of typical facial features, body asymmetry, clinodactyly or brachydactyly, genital abnormalities, café au lait spots, feeding difficulties or delayed speech. However, two women (subjects 1 and 3) had less specific features of down-turned corners of their mouth, and subject 1 also had retrognathia and a squeaky voice (Table 1 ). All women experienced early menarche (range, 10-11 yr) as compared with population standards [26]. Subjects 1 and 3 were diagnosed with polycystic ovary syndrome after menarche, defined as the presence of hirsutism [Ferriman \& Gallway score $>8$ ], oligomenorrhea (menstrual cycles $>45$ days), and increased testosterone, androstenedione and/or free androgen index (9). Insulin sensitivity (HOMA-IS \%) ranged from $18 \%$ to $65 \%$ and was thus well below normal limits in all subjects (reference mean \pm SEM from age-matched healthy volunteers [20]: $n=24$; age, $15.3 \pm 0.2 \mathrm{yr} ; 104 \% \pm 5$ ). 
Table 1 Clinical characteristics of women with low birthweight, precocious pubarche, short stature and insulin resistance

\begin{tabular}{|c|c|c|c|c|}
\hline Subject & 1 & 2 & 3 & 4 \\
\hline Age (yr) & 20 & 15 & 20 & 13 \\
\hline Gestational age (wk) & 39 & 38 & 40 & 37 \\
\hline Birthweight SDS (Kg) & $-3.9(1.8)$ & $-3.1(1.8)$ & $-3.0(2.2)$ & $-3.2(1.2)$ \\
\hline Birth length SDS $(\mathrm{cm})$ & $-3.9(42)$ & $-3.1(42)$ & $-3.0(45)$ & $-3.2(39)$ \\
\hline Head circumference SDS (cm) & $-1.8(31.5)$ & $-1.8(30.5)$ & $-2.5(31.0)$ & $\mathrm{n} / \mathrm{a}$ \\
\hline Mid-parental target height Z-score $(\mathrm{cm})$ & $-0.4(161)$ & $-0.8(159)$ & $-1.5(155)$ & $0.2(164)$ \\
\hline Adult height Z-score $(\mathrm{cm})$ & $-3.6(143)$ & $-2.1(153)$ & $-2.3(151)$ & $-2.3(151)$ \\
\hline Adult weight (Kg) & 39.3 & 56.0 & 68.0 & 41.7 \\
\hline Body mass index SDS $\left(\mathrm{Kg} / \mathrm{m}^{2}\right)$ & $-0.7(19.2)$ & $0.8(23.9)$ & $2.6(29.8)$ & $-1.0(18.3)$ \\
\hline Age at pubarche (yr) & 7.0 & 7.8 & 6.0 & 7.5 \\
\hline Age at menarche (yr) & 11.0 & 10.0 & 11.0 & 10.0 \\
\hline Polycystic ovary syndrome (age, yr) & 15.0 & No & 15.0 & No \\
\hline Body asymmetry & No & No & No & No \\
\hline Triangular shaped face & No & No & No & No \\
\hline Irregular crowded teeth & No & No & No & No \\
\hline Low set ears & No & No & No & No \\
\hline Retrognathia & Yes & No & No & No \\
\hline Prominent forehead & No & No & Yes & No \\
\hline Down-turned corners of the mouth & Yes & No & Yes & No \\
\hline Thin lips & No & No & No & No \\
\hline Squeaky voice & Yes & No & No & No \\
\hline Genital abnormalities & No & No & No & No \\
\hline Clinodactyly or brachydactyly & No & No & No & No \\
\hline Glucose (mg/dl) & $102 \uparrow$ & 88 & 81 & 83 \\
\hline Insulin $(\mathrm{mU} / \mathrm{L})^{\dagger}$ & $20.4 \uparrow$ & $17.9 \uparrow$ & $16.6 \uparrow$ & $17.4 \uparrow$ \\
\hline Insulin sensitivity $\left(\% \mathrm{HOMA}^{\dagger}{ }^{+}\right.$ & $65 \downarrow$ & $58 \downarrow$ & $54 \downarrow$ & $57 \downarrow$ \\
\hline 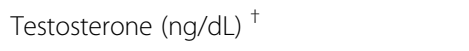 & $65 \uparrow$ & $64 \uparrow$ & $84 \uparrow$ & $60 \uparrow$ \\
\hline $\operatorname{SHBG}(\mu \mathrm{g} / \mathrm{dL})^{+}$ & $29 \uparrow$ & $31 \uparrow$ & $15 \uparrow$ & $27 \uparrow$ \\
\hline Free Androgen Index ${ }^{+}$ & $7.8 \uparrow$ & $7.2 \uparrow$ & $19.4 \uparrow$ & $7.7 \uparrow$ \\
\hline Androstenedione $(\mathrm{ng} / \mathrm{dL})^{\dagger}$ & $337 \uparrow$ & 190 & $567 \uparrow$ & $221 \uparrow$ \\
\hline DHEAS $(\mu \mathrm{g} / \mathrm{dL})^{\dagger}$ & $329 \uparrow$ & 86 & $311 \uparrow$ & 89 \\
\hline Karyotype & Normal & Normal & Normal & Normal \\
\hline IGF2 gene mutation & No & No & No & No \\
\hline H19 DMR \% (ref range $33.0-49.8)^{\#}$ & $2.2 \downarrow$ & 42.3 & 36.9 & 33.7 \\
\hline IGF2 DMRO\% (ref range $36.5-54.9)^{\#}$ & $27.6 \downarrow$ & 43.9 & 46.4 & 47.7 \\
\hline
\end{tabular}

SHBG, sex hormone-binding globulin; DHEAS, dehydroepiandrosterone-sulfate; free androgen index, testosterone $(\mathrm{nmol} / \mathrm{L}) \times \mathrm{SHBG}(\mathrm{nmol}(\mathrm{L}) / 100 ; \mathrm{n} / \mathrm{a}$, not available. ${ }^{+}$Reference data (mean \pm SEM) from age-matched healthy volunteers[20]: $n=24$; age, $15.3 \pm 0.2 \mathrm{yr}$; glucose: $79 \pm 1.8 \mathrm{mg} / \mathrm{dL}$; insulin: $11.3 \pm 1.0 \mathrm{mU} / \mathrm{L}$; insulin sensitivity (\% HOMA): $104 \pm 5$; testosterone: $31 \pm 3 \mathrm{ng} / \mathrm{dL} ;$ SHBG: $1.9 \pm 0.1 \mu \mathrm{g} / \mathrm{dL}$; free androgen index: $<5$; androstenedione: $156 \pm 14 \mathrm{ng} / \mathrm{dL}$; DHEAS: $125 \pm 12 \mu \mathrm{g} / \mathrm{dL}$. \# Reference range for $\%$ methylation, stated as $95 \%$ confidence interval derived from 95 control patients.

$\uparrow$ and $\downarrow$ are used in the table to indicate clearly increased and decreased laboratory values.

Subject 1 with the lowest birth weight SDS (-3.9) and shortest adult height of $143 \mathrm{~cm}(-3.6$ SDS) had severe H19 DMR hypomethylation: mean H19 DMR methylation was $2 \%$, compared to $95 \%$ confidence interval $33-49.8 \%$ methylation in 95 healthy controls, and moderately reduced IGF2 DMR0 methylation: $27.6 \%$ compared to 36.5-54.9\% in controls (Table 1 and Figure 1B). Subject 1 also had the highest fasting glucose which was in the impaired fasting glucose range, despite slim BMI $\left(19.2 \mathrm{~kg} / \mathrm{m}^{2}\right)$ and young adult age (20 years). 
All four subjects had a normal karyotype and no mutations in the IGF2 gene were detected.

\section{Discussion}

We describe the first case of severe hypomethylation of the IGF2-H19 DMR associated with severe pre-natal and post- natal growth restriction, insulin resistance, polycystic ovarian syndrome and precocious pubarche without convincing features of SRS. The majority of studies to date suggest $H 19$ DMR hypomethylation is a specific cause of SRS because it has not been found in any nonsyndromic SGA patients screened $[3,11,13,27,28]$. Many also report that the degree of hypomethylation is linked with severity of the phenotype [9,28-30]. However, there have been a few case reports of hypomethylation of H19 DMR associated with either hemi-hypertrophy alone $[29,30]$ or milder intrauterine growth restriction and post natal growth restriction associated with a prominent forehead and triangular facies as the only clinical signs [31]. Ours is the first case reported without asymmetry or abnormal face shape suggestive of SRS, however, she did have less specific features of retrognathia and a squeaky voice. Nonetheless, SRS is a disorder with marked clinical heterogeneity, and many of the clinical features regress with age.

We chose to study three molecular mechanisms (chromosomal, methylation and sequence abnormalities) by which IGF2 expression could have been reduced in 4 women selected to have a similar phenotype to our published case report [18] who had severe intrauterine growth restriction, precocious pubarche, short stature and insulin resistance, and was found to have a balanced translocation affecting her IGF2 gene regulation. We found 1 case with IGF2/H19 hypomethylation to a similar extent as observed in cases with SRS. The association of premature pubarche and insulin resistance in SRS has not been evaluated to date. Based on our observation from this study, we hypothesise that in addition to affecting pre- and post- natal growth, IGF2 in humans may have a causal role in timing of pubarche and insulin resistance. This remains to be evaluated in future studies involving patients with $I G F 2 / H 19$ hypomethylation.

The limitations of our analyses include the low sensitivity of routine karytoping in detecting small chromosomal rearrangements, and only exonic sequencing of the IGF2 gene. A full G-banded karyotype is able to detect structural abnormalities (deletions, translocations, inversions and insertions) at a resolution of approximately 10 million DNA base-pairs. Screening for mutations in the IGF2 gene including the intron-exon boundaries of all nine exons has not shown any abnormalities to date [32], hence is unlikely to be an important cause of IGF2 inactivation in humans.

\section{Conclusions}

Our case of growth restriction, premature pubarche and insulin resistance in the absence of body asymmetry or other features of SRS adds to the expanding phenotype of IGF2/H19 methylation abnormalities. Further studies are needed to confirm whether growth restriction in association with premature pubarche and insulin resistance is a specific manifestation of loss of IGF2 expression through IGF2/H19 loss of methylation.

\section{Abbreviations \\ BWS: Beckwith Wiedemann syndrome, CpG, cytosine guanine base pair; DMR: Differentially methylated region; DNA: Deoxyribonucleic acid; IGF2: Insulin like growth factor-2; SRS: Silver Russell syndrome; PCR: Polymerase chain reaction; SRS: Silver Russell syndrome.}

\section{Competing interests}

The authors declare that they have no competing interests.

\section{Authors' contributions}

RM conceived of the study design and drafted the manuscript; LI recruited the study cases, helped with the data analysis and interpretation; ATH participated in the study design and provided the control patient samples; JT performed the pyrosequencing analyses and helped in data analysis and interpretation. All authors read and approved the final manuscript.

\section{Acknowledgements}

$\mathrm{LI}$ is a Clinical Investigator of CIBERDEM (Centro de Investigación Biomédica en Red de Diabetes y Enfermedades Metabólicas Asociadas, Instituto de Salud Carlos III, Madrid, Spain). We sincerely thank all the patients who participated in this study.

\section{Author details}

${ }^{1}$ Faculty of Medical and Health Sciences, University of Auckland, Auckland Private Bag 92019, New Zealand. Endocrinology Unit, Hospital Sant Joan de Déu, University of Barcelona, Barcelona, and Centro de Investigación Biomédica en Red de Diabetes y Enfermedades Metabólicas Asociadas (CIBERDEM), ISCIII, Madrid, Spain. Institute of Biomedical and Clinical Sciences, Peninsula Medical School, Exeter, UK. ${ }^{4}$ Laboratory for Epigenetics, Centre National de Génotypage, CEA-Institut de Génomique, Evry, France.

Received: 15 December 2011 Accepted: 8 May 2012

Published: 30 May 2012

\section{References}

1. Weksberg $R$, et al: Beckwith-Wiedemann syndrome demonstrates a role for epigenetic control of normal development. Hum Mol Genet 2003, 12(1):R61-R68.

2. Azzi $S$, et al: Multilocus methylation analysis in a large cohort of $11 \mathrm{p} 15-$ related foetal growth disorders (Russell Silver and Beckwith Wiedemann syndromes) reveals simultaneous loss of methylation at paternal and maternal imprinted loci. Hum Mol Genet 2009, 18(24):4724-4733.

3. Netchine l, et al: 11 p15 imprinting center region 1 loss of methylation is a common and specific cause of typical Russell-Silver syndrome: clinical scoring system and epigenetic-phenotypic correlations. J Clin Endocrinol Metab 2007, 92(8):3148-3154.

4. Cui $\mathrm{H}$, et al: Loss of IGF2 imprinting: a potential marker of colorectal cancer risk. Science 2003, 299(5613):1753-1755.

5. Kaneda A, Feinberg AP: Loss of imprinting of IGF2: a common epigenetic modifier of intestinal tumor risk. Cancer Res 2005, 65(24):11236-11240.

6. Murrell A, Heeson S, Reik W: Interaction between differentially methylated regions partitions the imprinted genes lgf2 and $\mathrm{H} 19$ into parent-specific chromatin loops. Nature Genetics 2004, 36(8):889-893.

7. Ito $Y$, et al: Somatically acquired hypomethylation of IGF2 in breast and colorectal cancer. Hum Mol Genet 2008, 17(17):2633-2643.

8. Guo L, et al: Altered gene expression and methylation of the human chromosome 11 imprinted region in small for gestational age (SGA) placentae. Dev Biol 2008, 320(1):79-91. 
9. Gicquel $C$, et al: Epimutation of the telomeric imprinting center region on chromosome 11 p15 in Silver-Russell syndrome. Nature Genetics 2005, 37(9):1003-1007.

10. Engel $\mathrm{N}$, et al: Antagonism between DNA hypermethylation and enhancer-blocking activity at the H19 DMD is uncovered by CpG mutations. Nat Genet 2004, 36(8):883-888

11. Abu-Amero $S$, et al: Epigenetic signatures of Silver-Russell syndrome. J Med Genet 2010, 47(3):150-154.

12. Murrell A, et al: Distinct methylation changes at the IGF2-H19 locus in congenital growth disorders and cancer. PLoS One 2008, 3(3):e1849.

13. Bartholdi D, et al: Epigenetic mutations of the imprinted IGF2-H19 domain in Silver-Russell syndrome (SRS): results from a large cohort of patients with SRS and SRS-like phenotypes. J Med Genet 2009, 46(3):192-197.

14. DeChiara TM, Efstratiadis A, Robertson EJ: A growth-deficiency phenotype in heterozygous mice carrying an insulin-like growth factor II gene disrupted by targeting. Nature 1990, 345(6270):78-80.

15. Yakar S, Leroith D, Brodt $P$ : The role of the growth hormone/insulin-like growth factor axis in tumor growth and progression: Lessons from animal models. Cytokine Growth Factor Rev 2005, 16(4-5):407-420.

16. Fisher $A$, et al: Duplications of chromosome $11 \mathrm{p} 15$ of maternal origin result in a phenotype that includes growth retardation. Hum Genet 2002, 111:290-296.

17. Eggermann $T$, et al: Is maternal duplication of $11 \mathrm{p} 15$ associated with Silver-Russell syndrome? J Med Genet 2005, 42(5):e26.

18. Murphy $R$, et al: Severe intrauterine growth retardation and atypical diabetes associated with a translocation breakpoint disrupting regulation of the insulin-like growth factor 2 gene. J Clin Endocrinol Metab 2008, 93(11):4373-4380.

19. Ibanez $L$, et al: Clinical spectrum of premature pubarche: links to metabolic syndrome and ovarian hyperandrogenism. Rev Endocr Metab Disord 2009, 10(1):63-76.

20. Ibanez L, et al: Insulin sensitization for girls with precocious pubarche and with risk for polycystic ovary syndrome: effects of prepubertal initiation and postpubertal discontinuation of metformin treatment. J Clin Endocrinol Metab 2004, 89(9):4331-4337.

21. Knight B, Shields BM, Hattersley AT: The Exeter Family Study of Childhood Health (EFSOCH): study protocol and methodology. Paediatr Perinat Epidemiol 2006, 20(2):172-179.

22. Ferrandez-Longas $A$, et al: Estudio longitudinal de crecimiento $y$ desarrollo. In Patrones de crecimiento y desarrollo en España. Atlas de gráficas y tablas. Edited by Garcia-Dihinx A, Romo A, Ferrandez-Longas A. Madrid: ERGON; 2004:61-115

23. Ibanez $L$, et al: Insulin sensitization early after menarche prevents progression from precocious pubarche to polycystic ovary syndrome. J Pediatr 2004, 144(1):23-29.

24. Tost J, Gut IG: DNA methylation analysis by pyrosequencing. Nat Protoc 2007, 2(9):2265-2275.

25. Dejeux E, et al: Hypermethylation of the IGF2 differentially methylated region 2 is a specific event in insulinomas leading to loss-of-imprinting and overexpression. Endocr Relat Cancer 2009, 16(3):939-952

26. Carrascosa A, et al: [Secular growth changes. Weight, height and body mass index values in infant, children, adolescent and young adults from Barcelona population]. Med Clin (Barc) 2004, 123(12):445-451.

27. Schonherr $\mathrm{N}$, et al: (Epi)mutations in $11 \mathrm{p} 15$ significantly contribute to Silver-Russell syndrome: but are they generally involved in growth retardation? Eur J Med Genet 2006, 49(5):414-418.

28. Bruce $S$, et al: Clinically distinct epigenetic subgroups in Silver-Russell syndrome: the degree of $\mathrm{H} 19$ hypomethylation associates with phenotype severity and genital and skeletal anomalies. J Clin Endocrinol Metab 2009, 94(2):579-587.

29. Bliek J, et al: Hypomethylation of the $\mathrm{H} 19$ gene causes not only SilverRussell syndrome (SRS) but also isolated asymmetry or an SRS-like phenotype. Am J Hum Genet 2006, 78(4):604-614.

30. Zeschnigk M, et al: IGF2/H19 hypomethylation in Silver-Russell syndrome and isolated hemihypoplasia. Eur J Hum Genet 2008, 16(3):328-334
31. Eggermann T, et al: Broad clinical spectrum in Silver-Russell syndrome and consequences for genetic testing in growth retardation. Pediatrics 2009, 123(5):e929-e931.

32. Obermann C: Searching for genomic variants in IGF2 and CDKN1C in Silver-Russell syndrome patients. Mol Genet Metab 2004, 82:246-250.

doi:10.1186/1471-2350-13-42

Cite this article as: Murphy et al.: IGF2/H19 hypomethylation in a patient with very low birthweight, preocious pubarche and insulin resistance. BMC Medical Genetics 2012 13:42

\section{Submit your next manuscript to BioMed Central and take full advantage of:}

- Convenient online submission

- Thorough peer review

- No space constraints or color figure charges

- Immediate publication on acceptance

- Inclusion in PubMed, CAS, Scopus and Google Scholar

- Research which is freely available for redistribution 